\section{Review} Article

\title{
The Efficacy of FDG-PET for the Management of Esophageal Cancer: Review Article
}

\author{
Hiroyuki Kato, MD, PhD, FACS, and Masanobu Nakajima, MD, PhD
}

\begin{abstract}
${ }^{18}$ F-Fluorodeoxyglucose positron emission tomography has become an important informative modality during the past two decades. Because this type of tomography is a functional imaging construct, its primary use is in the field of oncology. It is being used increasingly in the management of several tumor types including esophageal cancer. These tomography scans can distinguish between benign and malignant tumors, identify stages of tumor spread, assess tumor recurrence, and monitor the response of malignant disease to therapy. The aim of this review was to outline the current and future roles of positron emission tomography in the management of esophageal cancer.
\end{abstract}

Keywords: esophageal cancer, FDG-PET, PET/CT, SUV, staging

\section{Introduction}

Esophageal cancer has proven to be one of the most difficult malignancies to cure despite improved surgical techniques, reduced perioperative mortality, and the introduction of multimodality therapy. ${ }^{1,2)}$ Accurate tumor staging - particularly with regard to the depth of tumor invasion, involvement of lymph nodes, and distant metastasis-is essential for optimal treatment selection and delivery. It facilitates individually tailored patient management. ${ }^{1)}$ Preoperative chemotherapy and chemoradiotherapy have been introduced as therapeutic options for locally advanced cancer to downsize the primary tumor. This step increases the rate of complete resection, which improves local tumor control and prevents the formation of distant metastases. ${ }^{3,4)}$

Perioperative mortality and morbidity are frequent in esophageal cancer patients. Thus, the use of neoadjuvant therapy is important as some patients have tumors that

$\overline{\text { Department of Surgery I, Dokkyo Medical University, Shimotsuga- }}$ gun, Tochigi, Japan

Received: February 28, 2012; Accepted: April 12, 2012

Corresponding author: Masanobu Nakajima, MD, PhD. Department of Surgery I, Dokkyo Medical University, 880 Kitakobayashi, Mibu, Shimotsuga-gun, Tochigi 321-0293, Japan Email: mnakajim@dokkyomed.ac.jp

(C)2012 The Editorial Committee of Annals of Thoracic and Cardiovascular Surgery. All rights reserved. show a good response to it and may even be cured without undergoing surgery. It is, therefore, important to establish a method that can distinguish patients who will have a partial response from those who will have a complete response to neoadjuvant therapy.

${ }^{18} \mathrm{~F}$-fluorodeoxyglucose positron emission tomography (FDG-PET), a noninvasive imaging modality, has been widely investigated during the past two decades in regard to its use in the management of esophageal cancer. ${ }^{5-15)}$ This imaging technique may facilitate definitive diagnoses in patients with malignant disease by differentiating between benign and malignant tumors, assessing extension of the disease, detecting tumor recurrence, and monitoring the response to therapy. ${ }^{16-18)}$

In this article, we review the role and usefulness of FDG-PET for disease staging and treatment of esophageal cancer.

\section{Imaging with PET or PET/CT}

Accurately staging esophageal carcinoma is of paramount importance. The prognosis and the treatment modalities and their sequences are highly variable, depending on the disease stage. FDG-PET is a promising modality for such accurate staging. FDG is the most common radiopharmaceutical used for PET imaging. It differentiates physiologically active tissues from malignant tumors based on enhanced glucose transport in the tumors. FDG 
(an analog of glucose) and glucose are taken up by cells via glucose transporters (GLUTs), which are located in cell membranes. The tendency of malignant cells to express abundant GLUT- ${ }^{19,20)}$ is the key to the amount of uptake. An abundance of these transporters may be a good predictor of the malignant potential of these cells and may correlate with the invasive potential of a tumor and the poor survival rates associated with esophageal cancer. ${ }^{21)}$

In the early days of FDG-PET application, ${ }^{7,22-24)}$ fusion imaging using PET and computed tomography (CT) was nonexistent. PET/CT is a relatively new imaging modality that can detect functional abnormalities. It is better than PET alone because the location of the FDG uptake is revealed by $\mathrm{CT}^{25)}$ Using this co-registration, motion artifacts are minimized, and the incidence of misregistration problems and diagnostic confusion is significantly decreased. The data acquired from PET images are reconstructed using standard reconstruction algorithms, which incorporate ordered subset expectation maximization. The data thus obtained may be analyzed quantitatively, semi quantitatively, or qualitatively. The most commonly used parameter for FDG uptake quantification is the standardized uptake value (SUV), which is the ratio of the injected radioactivity to total body weight.

\section{Staging of Esophageal Cancer}

\section{Primary lesion}

Endoscopy, endoscopic ultrasonography, esophagography, CT, and/or magnetic resonance imaging (MRI) have been used to determine the depth of tumor invasion. Recently, magnifying endoscopy has been used to determine tumor depth, as well. ${ }^{26,27)}$ It is difficult to judge the depth of invasion of esophageal cancer using FDG-PET. ${ }^{10,28,29)}$

Kato et al. reported the detection rate of primary tumor for each depth of invasion (T1-T4). ${ }^{10)}$ According to that report, the primary tumor was visualized by FDGPET in 119 of 149 patients (80\%). FDG uptake was detected in 21 of 49 patients with T1 tumors (43\%), 9 of 10 patients with T2 tumors (90\%), 50 of 51 patients with T3 tumors (98\%), and 39 of 39 patients with T4 tumors (100\%). Among the 81 patients who initially underwent surgery, FDG uptake was detected in 17 of the 40 who had pT1 tumors (43\%), in 3 of 17 (18\%) patients with pTla tumors (remaining within the muscularis mucosae), and in 14 of $23(61 \%)$ patients with pT1b tumors (involving the submucosa). FDG uptake rates in patients with pT2, pT3, and pT4 tumors were $83 \%, 97 \%$, and $100 \%$, respectively.
Based on these results, it cannot be clearly stated that FDG-PET is useful for detecting early esophageal cancer. Himeno et al. reported that pathological mucosal cancers were not detected by FDG-PET, whereas tumors that had invaded more deeply than the submucosal layer were detectable in all cases. Therefore, they stated that FDG-PET is useful for deciding if there was an indication for endoscopic resection. ${ }^{30)}$ In our recent study, the detection rate of mucosal cancer was poor, whereas submucosal (or deeper) cancers were detected in all cases (Fig. 1). At present, FDG-PET does not effectively determine tumor depth. It can be used, though, for tumor localization of advanced cancer.

\section{Lymph node metastasis}

Establishing the presence (or absence) of lymph node metastasis in patients with esophageal cancer is important because it affects the choice of treatment. A high frequency of lymph node metastasis is characteristic of esophageal cancer. Even when the tumor depth is at the submucosal level, lymph node metastasis is present in about $40 \%$ of patients. ${ }^{31)}$ The presence of lymph node metastasis, the extent of the metastatic field, and the number of metastatic lymph nodes influence the prognosis. Morphological imaging (e.g., by CT) evaluates the metastasis according to its size, morphology, and enhanced status, among other parameters. Morphological imaging cannot distinguish nonspecific lymph node swelling from metastatic swelling.

FDG-PET is able to diagnose a metastatic lymph node regardless of its size. Kato et al. reported that the minimum size of metastatic lesions that FDG-PET can detect is $6 \mathrm{~mm}$ diameter. ${ }^{10)}$ Thus, FDG-PET cannot detect micrometastases. There have been some reports of FDG accumulating in the presence of inflammatory lymphadenopathy. False-positive uptake by hilar lymph nodes is often observed, so diagnoses must be carefully constructed. ${ }^{11)}$

Kato et al. reported that FDG-PET showed 77.8\% sensitivity, $92.9 \%$ specificity, and $84.4 \%$ accuracy. They also showed that CT scanning had $61.1 \%$ sensitivity, $71.4 \%$ specificity, and $65.6 \%$ accuracy. ${ }^{10)}$ A meta-analysis that summed up the 12 reports concerning FDG-PET ${ }^{12)}$ found that the sensitivity, specificity, positive predictive value, and negative predictive value were $51 \%, 84 \%, 81 \%$, and $57 \%$, respectively. Other reports have demonstrated the superior performance of FDG-PET compared to CT. ${ }^{10,32)}$

Flamen et al. compared FDG-PET to endoscopic ultrasonography (EUS) in regard to diagnosing locoregional 
Table 1 Diagnostic accuracy of lymph node metastasis of esophageal cancer per lymph nodal group

\begin{tabular}{lccccc}
\hline & \multicolumn{4}{c}{ Lymphnodegroupaccuracy } \\
\cline { 2 - 5 } & Sensitivity & Specificity & Accuracy & PPV & NPV \\
\hline CT $(\mathrm{n}=117)$ & 26.5 & 97.5 & 91.3 & 50.1 & 93.2 \\
& $(45 / 170)$ & $(1722 / 1766)$ & $(1767 / 1936)$ & $(45 / 89)$ & $(1722 / 1847)$ \\
PET $(\mathrm{n}=117)$ & 32.9 & $98.9^{\mathrm{b}}$ & $93.1^{\mathrm{a}}$ & $74.7^{\mathrm{b}}$ & 93.9 \\
& $(56 / 170)$ & $(1747 / 1766)$ & $(1803 / 1936)$ & $(56 / 75)$ & $(1747 / 1861)$ \\
PET/CT (n= 50) & $46.0^{\mathrm{b}, \mathrm{d}}$ & $99.4^{\mathrm{c}}$ & $95.1^{\mathrm{c}, \mathrm{d}}$ & $87.0^{\mathrm{c}}$ & $95.5^{\mathrm{a}}$ \\
& $(40 / 87)$ & $(986 / 992)$ & $(1026 / 1079)$ & $(40 / 46)$ & $(986 / 1033)$ \\
\hline
\end{tabular}

PPV: positivepredictivevalue; NPV: negativepredictivevalue

${ }^{\mathrm{a} P}<0.05$ vs.CT; ${ }^{\text {}} \mathrm{P}<0.01$ vs.CT; ${ }^{\mathrm{c}} \mathrm{P}<0.001$ vs. $\mathrm{CT}$ and ${ }^{\mathrm{d}} \mathrm{P}<0.05$ vs.PET

lymph node metastasis. They found that the sensitivity of PET was $33 \%$, and that of EUS was $81 \%$; in contrast, the specificity of PET was $89 \%$, and that of EUS was $67 \%$. Concerning the diagnosis of regional lymph nodes and distant metastatic lymph nodes, the sensitivities of PET and CT + EUS were $43 \%$ and $48 \%$, respectively (not significant), whereas the specificities were $98 \%$ and $90 \%$, respectively (significant). ${ }^{33)}$ Based on these studies, the sensitivity of FDG-PET is not high for diagnosing lymph node metastasis, but the specificity is desirable. The differential diagnosis of metastatic lymph nodes versus inflammatory swelling lymph nodes is important for diminishing the false-positive and false-negative rates. Dual-time-point FDG-PET, which utilizes the difference in the maximum FDG uptake time by inflammation tissue versus cancer tissue, is a promising modality for solving this problem. ${ }^{34)}$

By adding FDG-PET to usual modalities such as CT and EUS, it is possible to improve diagnostic precision. Kato et al. proved that the diagnostic precision of FDGPET for diagnosing lymph node metastases is superior to that of CT (Table 1). ${ }^{35)}$

\section{Distant metastasis}

Distant metastasis is the most important factor that restricts therapeutic strategies. Distant metastases of esophageal cancer have been frequently observed in liver, lung, and bone, among other sites. FDG-PET is thought to be the most important modality in the diagnostic armamentarium for detecting distant metastatic disease in patients with esophageal cancer. In earlier studies, PET has revealed occult distant metastases at nodal and nonnodal sites in 5\%-40\% of patients ${ }^{36-38)}$ with moderate sensitivity $(\sim 67 \%)$ and high specificity $(\sim 97 \%) .{ }^{12)}$ It may also reveal osseous metastases that were not detected by conventional bone scintigraphy. ${ }^{39)}$ It has been reported that PET is superior to bone scintigraphy for detecting osteolytic lesions but not for osteoblastic lesions. ${ }^{40)}$ Using a logistic regression model in patients examined preoperatively with CT, EUS, and/or PET, one investigation found that PET was the only modality that predicted resection with curative intent because it excluded the presence of distant metastases. Those authors concluded that PET may be used to prevent unnecessary surgical exploration in patients who have M1 disease. ${ }^{41)}$ In another study, the use of FDG-PET changed the clinical management of 27 of 68 patients $(40 \%)$. In 12 patients, therapy was changed from curative to palliative (owing to detection of previously unsuspected distant metastases); in 3 patients, it was changed from palliative to curative; and in 12 patients, there was a change in the treatment modality or delivery but not in the treatment intent. ${ }^{42)}$

Distant metastasis is an important factor when determining if there is an indication for surgical therapy. FDGPET, which can search for lesions in the entire body with high specificity, is an essential modality.

\section{Diagnosis of Recurrent Disease}

The recurrence rate following radical therapy for esophageal cancer is high. Therefore, close follow-up is required. The usual imaging modalities (i.e., CT and MRI) often have some difficulty detecting a recurrent lesion because they detect abnormal lesions based on normal anatomy. FDG-PET is an extremely useful modality for detecting not only a locoregional recurrence but a distant metastatic recurrence (Fig. 2). ${ }^{13,43)}$ Kato et al. reported that the sensitivity, specificity, and accuracy of PET for diagnosing recurrence in locoregional lymph nodes were $100 \%, 75 \%$, and $84 \%$, respectively. The corresponding figures for $\mathrm{CT}$ were $84 \%, 86 \%$, and $85 \%$. These authors also reported that the sensitivity of PET is better than that of CT, but the specificity of CT is better than that of PET in regard to detecting distant organ re- 


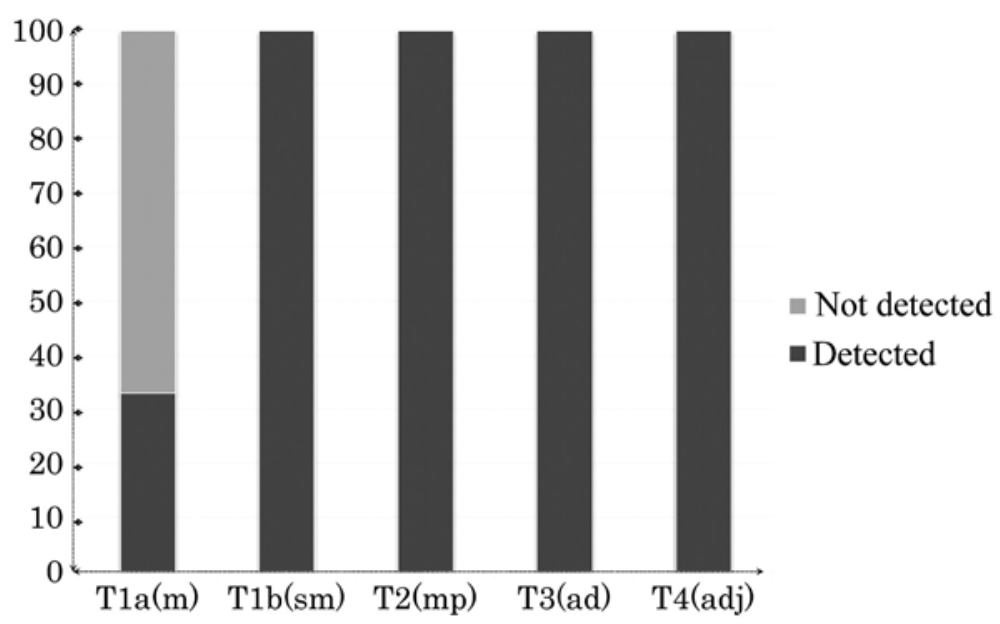

Fig. 1 primary esophageal cancer detection rates at each depth of invasion. The detection rate of tumors invading the mucosal layer (T1a) was 33\% and was $100 \%$ for tumors that invaded more deeply than the submucosal layer (T1b).
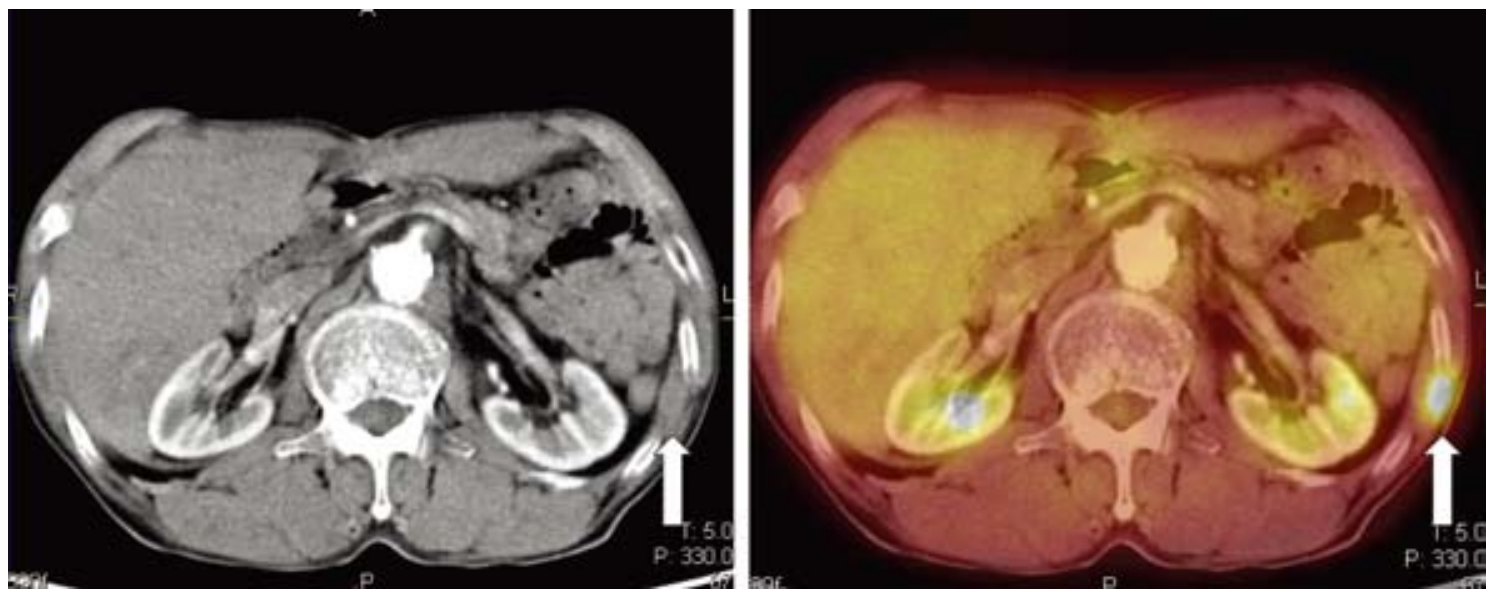

Fig. 2 Patient whose recurrent lesion was detected by ${ }^{18} \mathrm{~F}$-fluorodeoxyglucose positron emission tomography (FDG-PET).

He had undergone esophagectomy with lymphadenectomy 11 months before, and a thoracic wall recurrence was detected by FDG-PET. A: Computed tomography (CT) scan. It is difficult to identify the recurrent lesion (arrow). B: PET scan. A recurrent lesion is obvious at the left thoracic wall (arrow). Maximum standard uptake value (SUVmax) was 6.3.

currence. ${ }^{13)}$ The low specificity of PET was derived from false-positive FDG uptake in the reconstructed gastric tube, anastomosis, and hilar lymph nodes. It is improved by acquiring anatomical information using PET/CT.

\section{Evaluation of the Response of CRT}

For advanced esophageal cancer, neoadjuvant chemotherapy or chemoradiotherapy (CRT) is performed as a strategy to downstage (downsize) malignant lesions. ${ }^{44-50)}$
Among digestive malignancies, esophageal cancer is a promising candidate for a cure by CRT. ${ }^{51,52)}$ In some patients, the result of definitive CRT is equal to that achieved with a radical operation. ${ }^{53)}$ Patients whose tumors show a good response to neoadjuvant therapy may be cured without undergoing surgery. ${ }^{54)}$ Therefore, it is important to establish a method that can predict responders.

FDG-PET is useful for evaluating the response to CRT. CT and MRI cannot distinguish viable tumor tissues from fibrotic or necrotic residual tissues, whereas 

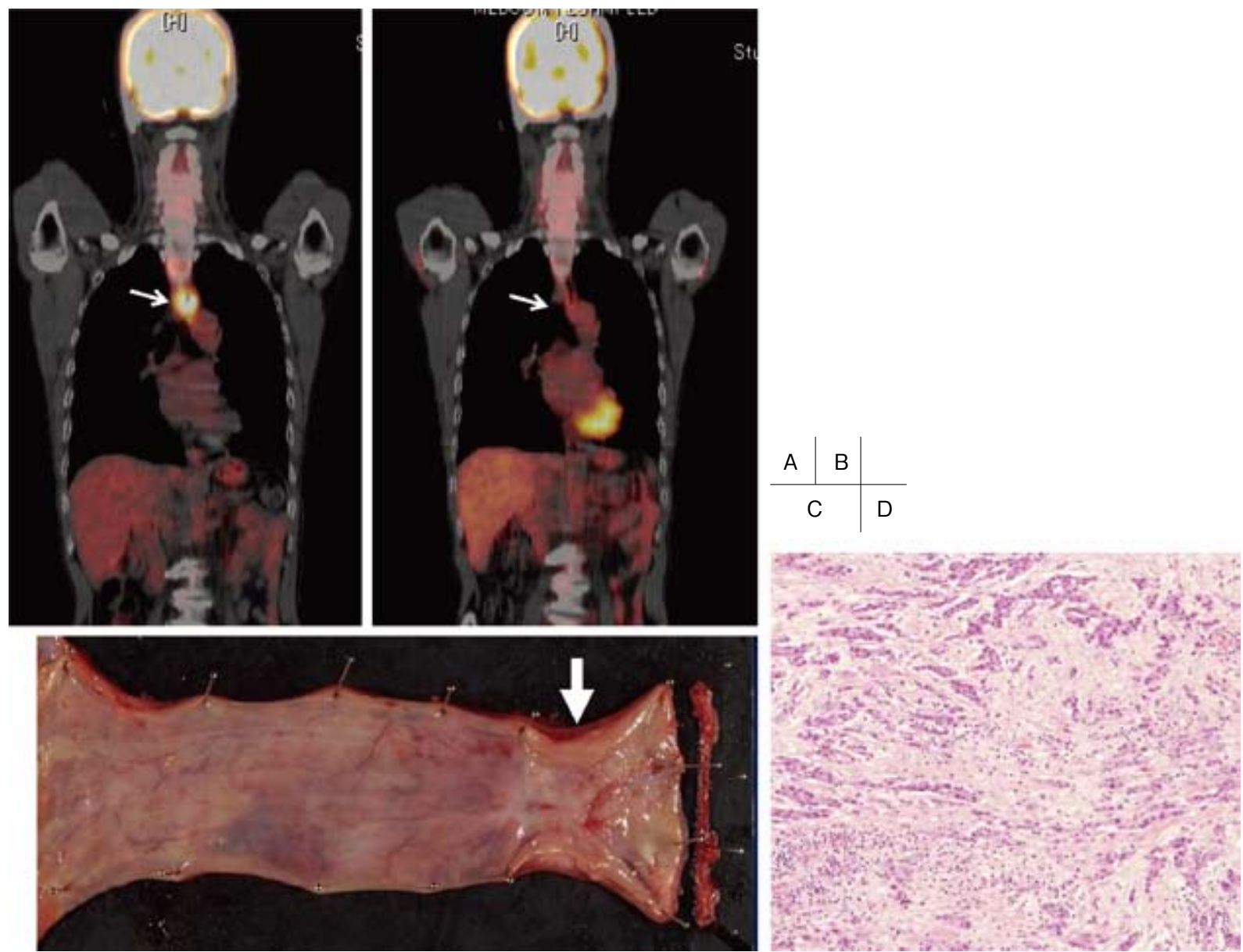

Fig. 3 PET scan of a patient rated as having a pathological partial response.

The scan was used as a monitor for neoadjuvant chemotherapy. A: The esophageal cancer demonstrated significant FDG uptake (arrow) (SUVmax 12.36) before neoadjuvant chemotherapy. B: Three weeks following chemotherapy, the FDG uptake was significantly decreased (arrow) (SUVmax 3.22). C: Subtotal esophagectomy was performed 4 weeks after finishing chemotherapy. The exposed tumor showed reduction (arrow). D: Pathology examination revealed a partial response (grade 1).

PET can perform a functional evaluation on the basis of metabolic information (Fig. 3). ${ }^{17)}$ The efficacy of FDGPET as a predictor of patients' responses to CRT has been reported in regard to esophageal cancer. ${ }^{18,55)}$ These studies discussed the effectiveness of PET evaluation by the rate at which the SUV was reduced. Pathological or clinical responders may be distinguished from nonresponders by establishing an optimal reduction rate. One study of adenocarcinoma of the esophagogastric junction noted that the change in FDG uptake 14 days after chemotherapy was significantly related to the rate at which the tumor volume was reduced. ${ }^{56)}$ There is some doubt about measuring the efficacy of CRT using a decrease in the SUV reduction rate. The SUV of FDG-PET reflects glucose metabolism. Therefore, reduction rates may be a good index of the pathological response but not the number of apoptotic cells. To begin to evaluate the number of residual viable tumor cells, the maximum SUV should be determined. Some studies have certified the importance of the FDG uptake value after induction or preoperative therapy. ${ }^{57,58)}$ Others have noted that the baseline SUV can predict the response to $\mathrm{CRT} .^{59,60)}$

Regardless of those findings, FDG-PET is a key modality for predicting the response and pathological efficacy of chemotherapy and CRT. We believe that in the near future FDG-PET will be active in predicting the response to molecular targeting therapy and immunotherapy.

\section{Summary}

FDG-PET is being increasingly used for diagnosing esophageal cancer and its initial staging. Its use in detecting 
recurrence during follow-up and monitoring the response to treatment is now standard practice. PET provides information that is complementary to that obtained by CT. The most important application of PET may be the selection of potential responders for various treatment modalities. Its survival benefit during multidisciplinary therapy for esophageal cancer is limited to those who display a pathological response. ${ }^{61-66)}$ Pathological responses can be seen only when the patient undergoes surgery, but FDGPET has the potential to predict an exact pathological response after induction therapy. That is, second-line therapy may be chosen based on FDG-PET results. The precision of FDG-PET and PET/CT needs improvement. Larger studies may better clarify whether there is indeed an incremental diagnostic improvement with FDG-PET.

In conclusion, FDG-PET is an important diagnostic modality in the therapeutic strategy for esophageal cancer. If higher-precision FDG-PET can be developed, it will contribute to improving the survival statistics for esophageal cancer patients. Also, with the identification of new FDG-PET tracers, we expect further expansion of the application of PET imaging in the field of esophageal cancer.

\section{Disclosure Statement}

Masanobu Nakajima and other co-authors have no conflict of interest.

\section{References}

1) Kato H, Fukuchi M, Miyazaki T, et al. Surgical treatment for esophageal cancer. Current issues. Dig Surg 2007; 24: 88-95.

2) Dehdashti F, Siegel BA. Neoplasms of the esophagus and stomach. Semin Nucl Med 2004; 34: 198-208.

3) Fink U, Stein HJ, Bochtler H, et al. Neoadjuvant therapy for squamous cell esophageal carcinoma. Ann Oncol 1994; 5: 17-26.

4) Raoul JL, Le Prisé E, Meunier B, et al. Neoadjuvant chemotherapy and hyperfractionated radiotherapy with concurrent low-dose chemotherapy for squamous cell esophageal carcinoma. Int J Radiat Oncol Biol Phys 1998; 42: 29-34.

5) Block MI, Patterson GA, Sundaresan RS, et al. Improvement in staging of esophageal cancer with the addition of positron emission tomography. Ann Thorac Surg 1997; 64: 770-6; discussion 776-7.

6) Luketich JD, Schauer PR, Meltzer CC, et al. Role of positron emission tomography in staging esophageal cancer. Ann Thorac Surg 1997; 64: 765-9.

7) Flanagan FL, Dehdashti F, Siegel BA, et al. Staging of esophageal cancer with $18 \mathrm{~F}$-fluorodeoxyglucose positron emission tomography. AJR Am J Roentgenol 1997; 168: 417-24.

8) Rankin SC, Taylor H, Cook GJ, et al. Computed tomography and positron emission tomography in the pre-operative staging of oesophageal carcinoma. Clin Radiol 1998; 53: 659-65.

9) Yeung HW, Macapinlac HA, Mazumdar M, et al. FDG-PET in Esophageal Cancer. Incremental Value over Computed Tomography. Clin Positron Imaging 1999; 2: 255-60.

10) Kato $H$, Kuwano $H$, Nakajima $M$, et al. Comparison between positron emission tomography and computed tomography in the use of the assessment of esophageal carcinoma. Cancer 2002; 94: 921-8.

11) Yoon YC, Lee KS, Shim YM, et al. Metastasis to regional lymph nodes in patients with esophageal squamous cell carcinoma: CT versus FDG PET for presurgical detection prospective study. Radiology 2003; 227: 764-70.

12) van Westreenen HL, Westerterp M, Bossuyt PM, et al. Systematic review of the staging performance of $18 \mathrm{~F}$ fluorodeoxyglucose positron emission tomography in esophageal cancer. J Clin Oncol 2004; 22: 3805-12.

13) Kato H, Miyazaki T, Nakajima M, et al. Value of positron emission tomography in the diagnosis of recurrent oesophageal carcinoma. Br J Surg 2004; 91: 1004-9.

14) Kato H, Nakajima M, Sohda M, et al. The clinical application of (18)F-fluorodeoxyglucose positron emission tomography to predict survival in patients with operable esophageal cancer. Cancer 2009; 115: 3196-203.

15) Hyun SH, Choi JY, Shim YM, et al. Prognostic value of metabolic tumor volume measured by $18 \mathrm{~F}$-fluorodeoxyglucose positron emission tomography in patients with esophageal carcinoma. Ann Surg Oncol 2010; 17: 115-22.

16) Brücher BL, Weber W, Bauer M, et al. Neoadjuvant therapy of esophageal squamous cell carcinoma: response evaluation by positron emission tomography. Ann Surg 2001; 233: 300-9.

17) Kato H, Kuwano H, Nakajima M, et al. Usefulness of positron emission tomography for assessing the response of neoadjuvant chemoradiotherapy in patients with esophageal cancer. Am J Surg 2002; 184: 279-83.

18) van Heijl M, Omloo JM, van Berge Henegouwen MI, et al. Fluorodeoxyglucose positron emission tomography for evaluating early response during neoadjuvant chemoradiotherapy in patients with potentially curable esophageal cancer. Ann Surg 2011; 253: 56-63.

19) Yen TC, See LC, Lai CH, et al. 18F-FDG uptake in squamous cell carcinoma of the cervix is correlated with glucose transporter 1 expression. J Nucl Med 2004; 45: 22-9.

20) Smith TA. FDG uptake, tumour characteristics and response to therapy: a review. Nucl Med Commun 1998; 19: $97-105$.

21) Kato H, Takita J, Miyazaki T, et al. Correlation of 
18-F-fluorodeoxyglucose (FDG) accumulation with glucose transporter (Glut-1) expression in esophageal squamous cell carcinoma. Anticancer Res 2003; 23: 3263-72.

22) Yasuda S, Raja S, Hubner KF. Application of wholebody positron emission tomography in the imaging of esophageal cancer: report of a case. Surg Today 1995; 25: 261-4.

23) Fukunaga T, Okazumi S, Koide Y, et al. Evaluation of esophageal cancers using fluorine-18-fluorodeoxyglucose PET. J Nucl Med 1998; 39: 1002-7.

24) Rice TW. Clinical staging of esophageal carcinoma. CT, EUS, and PET. Chest Surg Clin N Am 2000; 10: 471-85.

25) Karaosmano lu AD, Blake MA. Applications of PETCT in patients with esophageal cancer. Diagn Interv Radiol. 2012; 18: 171-82.

26) Kaga M, Inoue H, Kudo SE, et al. Microvascular architecture of early esophageal neoplasia. Oncol Rep 2011; 26: 1063-7.

27) Arima M, Tada M, Arima H. Evaluation of microvascular patterns of superficial esophageal cancers by magnifying endoscopy. Esophagus 2005; 2: 191-7.

28) Kato H, Miyazaki T, Nakajima M, et al. The incremental effect of positron emission tomography on diagnostic accuracy in the initial staging of esophageal carcinoma. Cancer 2005; 103: 148-56.

29) Kato H, Fukuchi M, Miyazaki T, et al. Positron emission tomography in esophageal cancer. Esophagus 2005; 2: 111-21.

30) Himeno S, Yasuda S, Shimada H, et al. Evaluation of esophageal cancer by positron emission tomography. Jpn J Clin Oncol 2002; 32: 340-6.

31) Endo M, Yoshino K, Kawano T, et al. Clinicopathologic analysis of lymph node metastasis in surgically resected superficial cancer of the thoracic esophagus. Dis Esophagus 2000; 13: 125-9.

32) van Vliet EP, Heijenbrok-Kal MH, Hunink MG, et al. Staging investigations for oesophageal cancer: a metaanalysis. Br J Cancer 2008; 98: 547-57.

33) Flamen P, Lerut A, Van Cutsem E, et al. Utility of positron emission tomography for the staging of patients with potentially operable esophageal carcinoma. J Clin Oncol 2000; 18: 3202-10.

34) Hu Q, Wang W, Zhong X, et al. Dual-time-point FDG PET for the evaluation of locoregional lymph nodes in thoracic esophageal squamous cell cancer. Eur J Radiol 2009; 70: 320-4.

35) Kato H, Kimura H, Nakajima M, et al. The additional value of integrated PET/CT over PET in initial lymph node staging of esophageal cancer. Oncol Rep 2008; 20: 857-62.

36) Meyers BF, Downey RJ, Decker PA, et al. The utility of positron emission tomography in staging of potentially operable carcinoma of the thoracic esophagus: results of the American College of Surgeons Oncology Group Z0060 trial. J Thorac Cardiovasc Surg 2007; 133: 738-45.
37) Blackstock AW, Farmer MR, Lovato J, et al. A prospective evaluation of the impact of 18-F-fluoro-deoxyD-glucose positron emission tomography staging on survival for patients with locally advanced esophageal cancer. Int J Radiat Oncol Biol Phys 2006; 64: 455-60.

38) Luketich JD, Friedman DM, Weigel TL, et al. Evaluation of distant metastases in esophageal cancer: 100 consecutive positron emission tomography scans. Ann Thorac Surg 1999; 68: 1133-6; discussion 1136-7.

39) Kato H, Miyazaki T, Nakajima M, et al. Comparison between whole-body positron emission tomography and bone scintigraphy in evaluating bony metastases of esophageal carcinomas. Anticancer Res 2005; 25: 4439-44.

40)Cook GJ, Houston S, Rubens R, et al. Detection of bone metastases in breast cancer by 18FDG PET: differing metabolic activity in osteoblastic and osteolytic lesions. J Clin Oncol 1998; 16: 3375-9.

41) van Westreenen HL, Heeren PA, van Dullemen HM, et al. Positron emission tomography with F-18-fluorodeoxyglucose in a combined staging strategy of esophageal cancer prevents unnecessary surgical explorations. J Gastrointest Surg 2005; 9: 54-61.

42) Duong CP, Demitriou H, Weih L, et al. Significant clinical impact and prognostic stratification provided by FDG-PET in the staging of oesophageal cancer. Eur J Nucl Med Mol Imaging 2006; 33: 759-69.

43) Flamen P, Lerut A, Van Cutsem E, et al. The utility of positron emission tomography for the diagnosis and staging of recurrent esophageal cancer. J Thorac Cardiovasc Surg 2000; 120: 1085-92.

44) Wolfárd A, Paszt A, Szentpáli K, et al. Efficacy and drawbacks of neoadjuvant chemoradiotherapy in squamous cell carcinoma of the thoracic esophagus. Hepatogastroenterology 2011; 58: 1214-9.

45) Miyata H, Yamasaki M, Kurokawa Y, et al. Multimodal treatment for resectable esophageal cancer. Gen Thorac Cardiovasc Surg 2011; 59: 461-6.

46)Jatoi A, Soori G, Foster NR, et al. Phase II study of preoperative pemetrexed, carboplatin, and radiation followed by surgery for locally advanced esophageal cancer and gastroesophageal junction tumors. J Thorac Oncol 2010; 5: 1994-8.

47) Okines A, Sharma B, Cunningham D. Perioperative management of esophageal cancer. Nat Rev Clin Oncol 2010; 7: 231-8.

48) Wijnhoven BP, van Lanschot JJ, Tilanus HW, et al. Neoadjuvant chemoradiotherapy for esophageal cancer: a review of meta-analyses. World J Surg 2009; 33: 2606-14.

49) Higuchi K, Koizumi W, Tanabe S, et al. Current management of esophageal squamous-cell carcinoma in Japan and other countries. Gastrointest Cancer Res 2009; 3: 153-61.

50) Kato H, Fukuchi M, Manda R, et al. The effectiveness of planned esophagectomy after neoadjuvant chemoradiotherapy for advanced esophageal carcinomas. Anticancer Res 2004; 24: 4091-6. 
51) Suzuki A, Xiao L, Hayashi Y, et al. Nomograms for prognostication of outcome in patients with esophageal and gastroesophageal carcinoma undergoing definitive chemoradiotherapy. Oncology 2012; 82: 108-13.

52) Morita M, Kumashiro R, Hisamatsu Y, et al. Clinical significance of salvage esophagectomy for remnant or recurrent cancer following definitive chemoradiotherapy. J Gastroenterol 2011; 46: 1284-91.

53) Kato K, Muro K, Minashi K, et al. Phase II study of chemoradiotherapy with 5-fluorouracil and cisplatin for Stage II-III esophageal squamous cell carcinoma: JCOG trial (JCOG 9906). Int J Radiat Oncol Biol Phys 2011; 81: 684-90.

54) Stahl M, Stuschke M, Lehmann N, et al. Chemoradiation with and without surgery in patients with locally advanced squamous cell carcinoma of the esophagus. J Clin Oncol 2005; 23: 2310-7.

55) Jingu K, Kaneta T, Nemoto K, et al. (18)F-fluorodeoxyglucose positron emission tomography immediately after chemoradiotherapy predicts prognosis in patients with locoregional postoperative recurrent esophageal cancer. Int J Clin Oncol 2010; 15: 184-90.

56) Wieder HA, Beer AJ, Lordick F, et al. Comparison of changes in tumor metabolic activity and tumor size during chemotherapy of adenocarcinomas of the esophagogastric junction. J Nucl Med 2005; 46: 2029-34.

57)Flamen $P$, Van Cutsem E, Lerut $A$, et al. Positron emission tomography for assessment of the response to induction radiochemotherapy in locally advanced oesophageal cancer. Ann Oncol 2002; 13: 361-8.

58) Ott K, Fink U, Becker K, et al. Prediction of response to preoperative chemotherapy in gastric carcinoma by metabolic imaging: results of a prospective trial. J Clin Oncol 2003; 21: 4604-10.

59) Hatt M, Visvikis D, Pradier O, et al. Baseline ${ }^{18}$ F-FDG
PET image-derived parameters for therapy response prediction in oesophageal cancer. Eur J Nucl Med Mol Imaging 2011; 38: 1595-606.

60) Suzuki A, Xiao L, Hayashi Y, et al. Prognostic significance of baseline positron emission tomography and importance of clinical complete response in patients with esophageal or gastroesophageal junction cancer treated with definitive chemoradiotherapy. Cancer 2011; 117: 4823-33.

61) Mandard AM, Dalibard F, Mandard JC, et al. Pathologic assessment of tumor regression after preoperative chemoradiotherapy of esophageal carcinoma. Clinicopathologic correlations. Cancer 1994; 73: 2680-6.

62) Hosoya Y, Shibusawa H, Nagai H, et al. Preoperative chemotherapy for advanced esophageal cancer and relation with histological effect. Surg Today 1999; 29: 689-94.

63) Brücher BL, Stein HJ, Zimmermann F, et al. Responders benefit from neoadjuvant radiochemotherapy in esophageal squamous cell carcinoma: results of a prospective phase-II trial. Eur J Surg Oncol 2004; 30: 963-71.

64) Berger AC, Farma J, Scott WJ, et al. Complete response to neoadjuvant chemoradiotherapy in esophageal carcinoma is associated with significantly improved survival. J Clin Oncol 2005; 23: 4330-7.

65) Saeki H, Morita M, Nakashima Y, et al. Neoadjuvant chemoradiotherapy for clinical stage II-III esophageal squamous cell carcinoma. Anticancer Res 2011; 31: 3073-7.

66) Scheer RV, Fakiris AJ, Johnstone PA. Quantifying the benefit of a pathologic complete response after neoadjuvant chemoradiotherapy in the treatment of esophageal cancer. Int J Radiat Oncol Biol Phys 2011; 80: 996-1001. 